\title{
Validation du facteur de correction de l'auto absorption des particules alpha dans une eau : application à la surveillance de sites spécifiques de la défense
}

\author{
A. CAZOULAT ${ }^{1}$, Y. LECOMPTE $^{1}$, S. BOHAND ${ }^{1}$, P. GÉRASIMO $^{1}$
}

(Manuscrit reçu le 18 juin 2006, accepté le 27 octobre 2006)

RÉSUMÉ La prévention d'une contamination interne chronique des populations par des radionucléides présents dans l'eau de boisson nécessite la mise en place d'une surveillance du niveau de radioactivité de l'eau. Le code de la santé publique introduit quatre paramètres pour la surveillance de la qualité radiologique des eaux destinées à la consommation humaine. Dans le cas particulier des émetteurs de particules alpha, compte tenu des caractéristiques de ces rayonnements, l'expression de l'activité alpha globale dans une eau nécessite au préalable de déterminer expérimentalement le facteur de correction de l'auto absorption de ces particules par le résidu sec de l'échantillon à analyser, au risque de sous estimer le résultat. Cet article décrit le protocole appliqué par le laboratoire du Service de protection radiologique des armées pour exprimer ce facteur $f$ en fonction de la masse $m$ de résidus, pour valider statistiquement cette relation et évaluer l'incertitude qui lui est associée. La relation obtenue dans l'exemple présenté est linéaire et s'exprime par $f=0,0253 m+1,2813$. Cette formule est valable pour des eaux de caractéristiques similaires au site pour lequel elle a été établie et pour des masses de résidus comprises entre 0 et $100 \mathrm{mg}$. L'incertitude relative sur $f$ quelle que soit la masse de résidu est de $11 \%(k=2)$. Dans un deuxième temps sont présentés à titre d'illustration les sites de la défense qui nécessitent une surveillance de l'activité alpha dans l'eau, comme les sites d'expérimentation des obus flèches à l'uranium appauvri de Bourges et de Gramat.

ABSTRACT Self-absorption alpha particle factor validation in water: Interest in the monitoring of specific military sites.

The population internal intake prevention by radionuclides present in water needs to monitor the radioactive level of this water. The French public health legislation introduces four radiological parameters for monitoring water, such as the gross alpha radioactivity. Regarding the alpha particle characteristics, a self-absorption factor has to be established beforehand, not to underestimate the real alpha radioactivity in water samples. The aim of this paper is to describe the procedure used by the laboratory of the French army radioprotection service to determine this $f$ factor, which depends on the water residue mass $m$ after evaporation. The relation is $f=0.0253 m+1.2813$. This formula can be employed for such waters used in this experiment and for masses between 0 and $100 \mathrm{mg}$. The uncertainty associated is about $11 \%(k=2)$. Some water monitoring examples are given. It is specially the case of depleted uranium shells experiment centres, localized in Gramat and Bourges.

Keywords: water / alpha counting / self-absorption / monitoring sites

${ }^{1}$ Service de protection radiologique des armées (SPRA), Laboratoire de contrôle radiotoxicologique, 1 bis rue du lieutenant Raoul Batany, 92141 Clamart Cedex, France. 


\section{Introduction}

La prévention d'une contamination interne chronique des populations par des radionucléides présents dans l'eau de boisson nécessite la mise en place d'une surveillance du niveau de radioactivité de l'eau. Des indicateurs pour la qualité radiologique des eaux destinées à la consommation humaine ont été introduits par l'Organisation mondiale de la santé (OMS), repris dans une directive européenne puis finalement dans le code de la santé publique en décembre 2001 (Gérasimo et Laroche, 2004). Parmi ces indicateurs, l'activité alpha globale de l'eau est adaptée à la mise en évidence d'une contamination par les radio-isotopes émetteurs de particules alpha. La méthode normalisée pour déterminer cette activité passe par l'évaporation complète de l'échantillon d'eau et fait appel à des appareils de mesure de la radioactivité alpha. Le phénomène d'auto absorption de ces particules au sein du résidu est une interférence qu'il est nécessaire d'évaluer pour chaque échantillon afin de déterminer avec précision son activité.

Cet article décrit dans un premier temps le protocole proposé par le laboratoire du Service de protection radiologique des armées (SPRA) pour évaluer cette auto absorption en fonction de la masse de résidu sec et pour valider statistiquement sa détermination. Dans un deuxième temps sont présentés à titre d'illustration les sites de la défense dans lesquels a été mis en place une surveillance des eaux et qui nécessitent l'usage de cette relation. C'est le cas des sites d'expérimentation des obus flèches à l'uranium appauvri, élément émetteur alpha concentré sous forme de métal et susceptible de contaminer l'eau des nappes phréatiques.

\section{Les indicateurs de la qualité radiologique des eaux}

Le code de la santé publique CSP (2002) introduit quatre indicateurs pour la qualité radiologique des eaux destinées à la consommation humaine : l'activité en tritium, l'activité alpha globale, l'activité bêta globale résiduelle, toutes exprimées en Becquerel par litre ( $\mathrm{Bq} \mathrm{L}^{-1}$ ), et la dose totale indicative (ou DTI), exprimée en millisievert par an $\left(\mathrm{mSv} \mathrm{an}^{-1}\right)$. Ces indicateurs permettent de détecter la présence anormale de radionucléides, artificiels ou naturels, du fait d'un rejet éventuel d'effluents radioactifs imputables à une activité industrielle ou à un accident, ou permettent de connaître le profil radiologique d'une eau (activité naturelle élevée).

Les activités alpha et bêta globales permettent d'évaluer en première intension la dose totale indicative (DTI). Cette notion, introduite par la directive 98/83/CE (JOEC, 1998) relative à la qualité des eaux destinées à la consommation humaine et définie par arrêté (JORF, 2004), représente la dose efficace engagée résultant d'une incorporation, pendant un an, de tous les radionucléides naturels et artificiels détectés dans une distribution d'eau (à l'exclusion du radon et de ses descendants 
à vie courte). Conformément aux recommandations de l'OMS (WHO, 2004), la valeur guide de la dose efficace engagée attribuable à la consommation d'eau est fixée à $0,1 \mathrm{mSv}$ par an. Pour respecter ce seuil, les valeurs guides de la réglementation française pour les activités alpha et bêta globales sont respectivement $0,1 \mathrm{~Bq} \mathrm{~L}^{-1}$ et $1 \mathrm{~Bq} \mathrm{~L}^{-1}$ (JORF, 2004). Au-delà de ces valeurs, $l^{\prime}$ identification et la quantification des radio-isotopes naturels et artificiels doivent être effectuées et la DTI calculée conformément à l'arrêté du $1^{\text {er }}$ septembre 2003 définissant les modalités de calcul des doses efficaces et des doses équivalentes résultant de l'exposition des personnes aux rayonnements ionisants (JORF, 2003a).

Les isotopes constitutifs de l'uranium appauvri étant tous émetteurs de particules alpha, la mesure de l'activité alpha globale de l'eau est un moyen simple et efficace pour la surveillance en routine d'une eau susceptible d'être contaminée par de l'uranium appauvri. Les méthodes d'analyse spécifiques de l'uranium dans l'eau (chromatographiques le plus souvent) ne seront mises en œuvre qu'en cas d'activité alpha globale supérieure aux valeurs guide. Ces méthodes sont plus sensibles mais aussi plus longues à mettre en œuvre.

\section{Mesure de l'activité alpha globale d'une eau}

La méthode normalisée pour l'analyse des émetteurs alpha dans une eau repose sur l'évaporation d'un échantillon d'eau dont la teneur en sels est inférieure à $2 \mathrm{~g} \mathrm{~L}^{-1}$, suivie de la mesure de la radioactivité du résidu sec (NF, 2004). Bien que des méthodes plus rapides et spécifiques, basées le plus souvent sur la scintillation liquide, aient été développées par ailleurs (Toscheva et al., 2004 ; Kleinschmidt Ross, 2004), c'est la méthode normalisée qui a été retenue par le SPRA.

\subsection{Mode opératoire}

La mesure de la radioactivité alpha global dans un échantillon d'eau consiste schématiquement à :

1. prélever un volume ou une masse d'eau exactement mesurés,

2. filtrer si nécessaire,

3. évaporer à sec l'eau brute et/ou l'eau filtrée sur coupelle inox et peser le résidu obtenu,

4. mesurer la radioactivité alpha à l'aide d'appareils type compteurs à gaz (comptage en mode proportionnel) ou solides (scintillateur au sulfure de zinc, $\mathrm{ZnS}$ ), préalablement étalonnés,

5. rapporter l'activité mesurée au volume prélevé (ou à la masse) selon la formule définie ci-après. 


\subsection{Calcul de l'activité}

D'après cette même norme, l'activité alpha globale s'exprime selon la formule :

$$
A=\frac{N-N_{0}}{t_{N} \times P E} \times \frac{100}{\rho} \times f
$$

avec :

- A : valeur de l'activité d'un mélange de radio-isotopes émetteurs alpha déterminée par un ensemble de mesures dont le rendement du compteur $\rho$ a été déterminé au moyen d'une source étalon de ${ }^{239} \mathrm{Pu}\left(\mathrm{ou}{ }^{241} \mathrm{Am}\right)$ dans les mêmes conditions géométriques. A s'exprime en becquerel par unité de masse ou de volume ;

- $N_{0}$ : mouvement propre, valeur indiquée par le dispositif de mesure de rayonnement, placé dans ses conditions normales d'emploi, en l'absence de la source dont on veut mesurer le rayonnement. $N_{0}$ s'exprime en coups ;

- $\quad N$ : nombre de coups détectés pendant le temps de comptage $t_{N}$ du résidu sec de l'échantillon à analyser, exprimé en secondes ;

- $\rho$ : rendement du compteur, rapport du nombre d'événements enregistrés pendant la durée de comptage à l'activité de la source et à la durée de ce comptage. $\rho$ s'exprime en pourcentage.

Remarque : la norme exprime $\rho$ par rapport à l'activité de la source. La pratique du laboratoire est de déterminer le rendement de chaque détecteur à l'aide du flux (mentionné sur le certificat d'étalonnage de la source étalon). Les expériences ayant toujours été réalisées à partir du flux, il n'y a pas d'incidence sur le résultat final.

- $f$ : facteur de correction de l'auto absorption ;

- $\quad P E$ est la masse (g) ou le volume (L) de la prise d'essai.

Cette formule est classiquement utilisée dans les laboratoires pour les mesures de radioactivité, à l'exception du facteur $f$ qui est spécifique de l'analyse des solutions aqueuses chargées en ions.

\section{Facteur de correction de l'auto absorption $f$}

\subsection{Description du phénomène}

Après évaporation sur coupelle d'un volume d'eau défini, le résidu minéral obtenu (contenant les émetteurs alpha à mesurer) est appelé par convention « source ».

Le taux de comptage mesuré par un instrument de mesure de radioactivité est directement lié aux rayonnements émis par la source et non à l'activité propre de 
cette source. Ce phénomène, lié à la perte de tout ou partie de l'énergie du rayonnement du fait des interactions avec les constituants de la source (auto absorption), doit être pris en compte au niveau de la mesure de l'activité alpha des échantillons. Il est en effet important de déterminer la relation entre le rendement des sources $\left(\varepsilon_{s}\right)$ issues de la préparation des échantillons à analyser et la masse du résidu d'évaporation, ce rendement dépendant en particulier de la charge en sels de la matrice déposée.

Remarque 1. Les particules alpha étant des particules lourdes, le phénomène de rétrodiffusion qui représente la diffusion par la source elle-même ou son matériau support du rayonnement émis par cette source vers le détecteur, est peu probable (à l'inverse des particules bêta).

Remarque 2. Le facteur de correction de l'auto absorption $f$ est défini dans la norme NF M 60-801 par « le rapport du nombre d'événements mesurés à l'origine du phénomène au nombre d'événements réellement détectables ». Il représente donc l'inverse d'un rendement de source $\varepsilon_{S}\left(f=1 / \varepsilon_{s}\right)$.

\subsection{Intérêts de la détermination du facteur $f$}

Un calcul d'activité alpha globale dans une eau sans appliquer un facteur de correction conduirait à une sous estimation du résultat, d'autant plus importante que la masse de résidus est élevée. Il est d'ailleurs évoqué aussi bien dans les normes françaises que par l'Agence américaine de protection de l'environnement en charge d'établir les règles de surveillance radiologique des eaux aux État-Unis (Parsa et al., 2005).

\section{Détermination de la relation entre le facteur $f$ et la masse de résidus $m$}

\subsection{Principe}

La mesure de l'activité d'une série de résidus d'évaporation d'eau de masse différente, surchargée par une quantité identique d'un traceur émetteur alpha d'activité volumique connue, permet de déterminer la relation entre le facteur de correction de l'auto absorption et la masse du résidu d'évaporation d'un échantillon d'eau.

\subsection{Matériel et méthode}

Le résidu d'évaporation d'une eau est constitué par l'ensemble des matières dissoutes dans cette eau. Il a été envisagé de constituer des résidus d'évaporation 
à partir de prises d'essai de $100 \mathrm{~mL}$ et $200 \mathrm{~mL}$ d'échantillons d'eaux prélevés en amont d'un site surveillé en routine par notre laboratoire. Ces eaux ont une composition minérale identique à celles des eaux analysées en aval du site et sont exemptes de toute contamination radioactive (exceptée la composante naturelle qui sera prise en compte dans les blancs). Les volumes de prise d'essai choisis répondent aux critères suivants :

- ils sont représentatifs de l'échantillon à analyser (le volume des prélèvements parvenant au laboratoire est de 1 à $2 \mathrm{~L}$ ),

- ils sont suffisamment faibles pour permettre une évaporation rapide,

- ils sont suffisamment grands pour garantir une limite de détection inférieure ou égale à $0,04 \mathrm{~Bq} / \mathrm{L}$ (JORF, 2003b).

D'après l'expérience acquise au laboratoire, la masse de résidus d'évaporation obtenue à partir de ces prises d'essai est comprise entre 20 et $100 \mathrm{mg}$.

\subsubsection{Préparation des échantillons}

Pour chaque échantillon d'eau, quatre prises d'essai ont été effectuées, deux de $100 \mathrm{~mL}$ et deux de $200 \mathrm{~mL}$. Pour chaque volume, seule une prise d'essai est tracée, l'autre servira de blanc. Tous les échantillons tracés doivent recevoir une quantité de traceur du même ordre de grandeur : le traçage est réalisé par ajout dans l'un des deux béchers d'une masse précise de ${ }^{239} \mathrm{Pu}$ d'activité connue. La pesée se fait par différence de masse. Connaissant la masse de traceur ajoutée dans le bécher et l'activité massique du traceur, l'activité $A$ contenue dans le bécher est donnée par :

$A(\mathrm{~Bq})=$ masse de traceur ajoutée $(\mathrm{g}) \times$ activité du traceur ${ }^{239} \mathrm{Pu}\left(\mathrm{Bq} \mathrm{g}^{-1}\right)$.

En pratique, la masse de traceur ajoutée est de l'ordre de $45 \mathrm{mg}$ et son activité massique de $0,9 \mathrm{~Bq} \mathrm{~g}^{-1}$, soit une activité introduite dans les échantillons de l'ordre de 40 à $50 \mathrm{mBq}$.

La balance utilisée à cette étape est une Sartorius ${ }^{\circledR}(e=1 \mathrm{mg}, d=0,01 \mathrm{mg})$.

\subsubsection{Phase d'évaporation}

$25 \mathrm{~mL}$ de $\mathrm{HNO}_{3}$ concentré sont ajoutés pour $100 \mathrm{~mL}$ de prise d'essai afin de limiter l'adsorption des émetteurs alpha sur les parois du contenant en les solubilisant. L'ensemble fait l'objet d'une évaporation douce sur plaque chauffante, sans porter à ébullition, jusqu'à l'obtention d'un volume d'environ $5 \mathrm{~mL}$. La température doit être aussi uniforme que possible sur l'ensemble de la plaque afin d'éviter les zones de « surchauffe » qui provoqueraient des pertes par projections. 
Pendant ce temps, des coupelles en tôle d'acier inoxydable embouti à rebord, de diamètre 4,7 cm sont identifiées sur leur face externe à l'aide d'un graveur, puis dégraissées par un mélange d'acétone et d'alcool absolu afin d'obtenir un dépôt uniforme sur la coupelle. Ces coupelles sont ensuite pesées sur une balance Voyageur $^{\circledR}(e=1 \mathrm{mg} ; d=0,1 \mathrm{mg})$.

Quand il ne reste environ que 4 à $5 \mathrm{~mL}$ de liquide dans les béchers, ils sont prélevés par fractions de 2 à $3 \mathrm{~mL}$ à l'aide d'une pipette à usage unique puis déposés en une couche fine et uniforme sur une coupelle en inox.

L'évaporation se termine sous lampe chauffante en porcelaine à une température inférieure à $65^{\circ} \mathrm{C}$. La température doit être aussi uniforme que possible.

Après refroidissement, la masse de résidus est mesurée à l'aide d'une balance Voyageur $^{\circledR}(e=1 \mathrm{mg} ; d=0,1 \mathrm{mg})$. La coupelle ainsi obtenue constitue la source.

\subsubsection{Mesurage de l'activité}

Le comptage s'effectue pendant 54000 secondes sur un compteur à scintillation au ZnS type alpha NU20 (Canberra $\left.{ }^{\circledR}\right)$. Le résultat obtenu est exprimé en nombre de coups.

\subsubsection{Calcul du facteur de correction de l'auto absorption}

En soustrayant, pour une masse de résidu donnée, le nombre de coups mesurés avec la «source» sans traceur (CST) au nombre de coups mesuré avec la « source » avec traceur (CAT), on obtient le nombre de coups dus au marqueur réellement détecté. C'est pourquoi, pour une masse donnée il est impératif de compter sur une même voie de comptage les échantillons donnant CST et CAT (chaque voie ayant un rendement spécifique)

$$
f=\frac{\text { activité ajoutée }}{C A T-C S T} \times \frac{\rho}{100} \times t
$$

$t$ est le temps de comptage en secondes (54000 secondes), $\rho$ est le rendement du compteur $(\%)$ calculé à l'aide du flux de la source étalon $\left({ }^{239} \mathrm{Pu}\right)$ sur la voie de comptage considérée et « activité déposée » (en Bq) est l'activité du traceur ${ }^{239} \mathrm{Pu}$ ajoutée dans l'échantillon.

\subsubsection{Cas particulier des faibles masses}

Les masses des résidus d'évaporation obtenus au cours de notre étude sont supérieures à $10 \mathrm{mg}$. Afin d'évaluer la valeur de $f$ pour les faibles masses de 


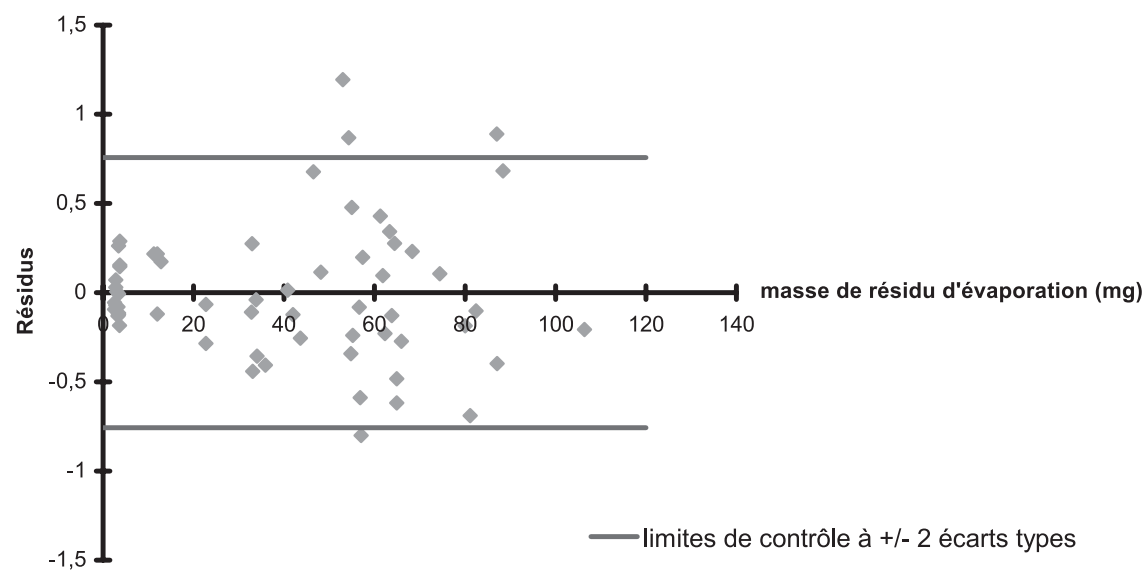

Figure 1. Graphique des résidus utilisé pour déterminer les valeurs aberrantes.

Graphic of residus used to get absurd values.

résidus d'évaporation, nous avons étudié les résultats obtenus avec les blancs de manipulation et avec des témoins de rendement : les deux sont constitués par des prises d'essai de $100 \mathrm{~mL}$ d'eau désionisée et les témoins de rendement sont tracés avec la solution de ${ }^{239} \mathrm{Pu}$.

Ces échantillons sont traités selon le mode opératoire décrit précédemment et le facteur $f$ déterminé selon la même formule.

\subsection{Résultats et discussion}

Les essais sur les eaux analysées $(100 \mathrm{~mL}$ et $200 \mathrm{~mL})$ et l'eau désionisée ont permis de collecter 60 résultats expérimentaux avec des masses de résidus comprises entre 2,5 mg et 106,4 mg. $f$ a été calculé suivant la formule (1) et une relation linéaire entre $f$ et $m$ a été établie par la méthode des moindres carrés à l'aide du tableur Excel ${ }^{\circledR}$.

\subsubsection{Détermination des valeurs aberrantes}

Compte tenu du nombre relativement important d'échantillons, une recherche des valeurs aberrantes à l'aide du graphique de variation des résidus a été effectuée (Fig. 1). Cette méthode simple a été décrite dans un ouvrage de l'agence française de normalisation (Souvey, 2002) et retenue ici pour notre étude. On appelle résidu la distance d'un point expérimental à la droite de régression (distance verticale entre le point observé et le point correspondant sur la droite de régression). Les limites de contrôles choisies sont égales à deux fois l'écart-type des résidus. 


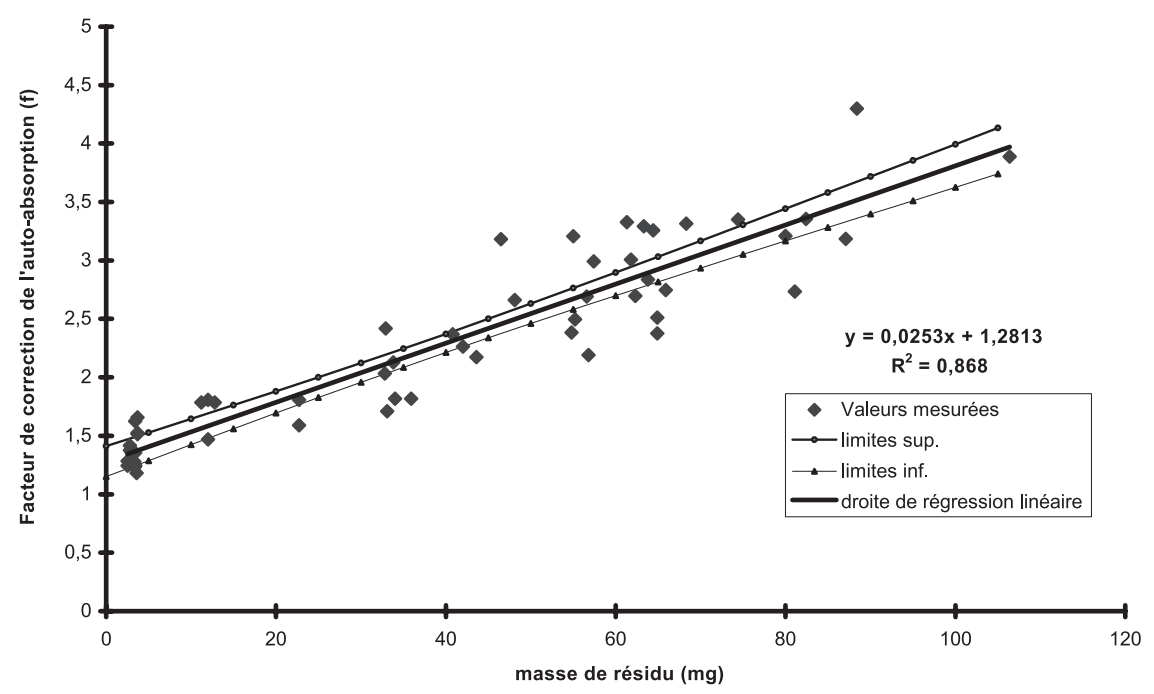

Figure 2. Représentation graphique du facteur de correction de l'auto absorption $f$ en fonction de la masse de résidus d'évaporation $m$ et représentation de son intervalle de confiance (après exclusion des valeurs aberrantes).

Graphic representation of self-absorption alpha particle factor $f$ as a function of the residues mass $m$ after evaporation and representation of its interval of acceptation (after absurd values exclusion).

Le point dont le résidu est en dehors de ces limites est considéré comme aberrant. La régression linéaire entre le facteur $f$ et la masse $m$ du résidu d'évaporation est ensuite recalculée en excluant ces données. Dans notre cas, quatre résultats aberrants ont ainsi été exclus.

\subsubsection{Relation entre f et $m$}

La relation obtenue entre $f$ et $m$ pour l'intervalle des masses comprises entre 0 et $106,4 \mathrm{mg}$ et après élimination des 4 valeurs aberrantes est de type $f=0,0253 \mathrm{~m}+$ 1,2813 . Cette droite est représentée en figure 2.

\subsubsection{Validation de la droite de régression}

La validation statistique de cette droite a été envisagée selon le modèle décrit par Bouyer (2000) et Souvey (2002). 


\subsubsection{Notations et formules de calcul}

\begin{tabular}{cc|}
\hline Caractéristiques & Notation \\
\hline Taille de l'échantillon & $n$ \\
\hline Ordonnée à l'origine de la droite de régression & $a$ \\
\hline Coefficient directeur de la droite de régression & $b$ \\
Moyenne sur $x$ (masse des résidus) & $\bar{x}$ \\
Moyenne sur $y$ (valeurs de $f$ ) & $\bar{y}$ \\
Variance de $x$ & $S x^{2}$ \\
Variance de $y$ & $S y^{2}$ \\
Variance résiduelle & $S r^{2}=\frac{n-1}{n-2}\left(S y^{2}-b^{2} S x^{2}\right)$ \\
\hline
\end{tabular}

\subsubsection{Test de la pente de la droite de la régression}

Le test de la pente de la droite de régression a pour objectif de montrer que cette pente est significativement différente de zéro au risque $\alpha$ près. Ce test consiste à calculer $t_{0}$ ci-dessous et à le comparer à la valeur seuil au risque $\alpha / 2$ de la loi de Student à $n-2$ degré de liberté (ddl)

$$
t_{0}=\frac{b}{\sqrt{S b^{2}}}
$$

avec :

$b:$ coefficient directeur (pente) de la droite de régression,

$S b^{2}:$ variance de la pente et $S b^{2}=\frac{S r^{2}}{S x^{2}(n-1)}$.

On obtient $t_{0}=18,84$ à $54 \mathrm{ddl}:$ le test est significatif avec un degré de signification $p<0,001$.

Le coefficient directeur de la droite de régression est significativement différent de zéro $(\alpha=5 \%)$ : il y a bien une relation entre la valeur du coefficient de correction de l'auto absorption $f$ et la masse du résidu d'évaporation que la droite de régression linéaire permet de modéliser.

\subsubsection{Test de l'ordonnée à l'origine de la droite de régression}

Ce test a pour objet de vérifier si l'ordonnée à l'origine de la régression est significativement différente de zéro au risque $\alpha$ près. Ce test consiste à calculer $t_{0}$ 
ci-dessous et à le comparer à la valeur seuil au risque $\alpha / 2$ de la loi de Student à $n-2$ ddl

$$
t_{\text {test }}=\frac{0-a}{\sqrt{\left[\frac{1}{n}-\frac{\bar{x}^{2}}{\Sigma(x-\bar{x})^{2}}\right] S r^{2}}}
$$

On obtient $t_{0}=19,71$ à $54 \mathrm{ddl}:$ le test est significatif $(p<0,001)$. L'ordonnée à l'origine de la droite de régression est significativement différente de zéro $(\alpha=$ $5 \%$ ). Ce constat est en accord avec les propriétés physiques des particules alpha : même sans résidu d'évaporation, le phénomène d'auto absorption des particules alpha est significatif.

\subsubsection{Détermination de l'intervalle de confiance de la droite de régression et des incertitudes sur la valeur def}

Le calcul de l'incertitude du résultat final nécessite de connaître l'incertitude sur le facteur de correction de l'auto absorption $f$. Pour une valeur de masse de résidu $x_{0}$, l'intervalle de confiance de la valeur $f(y)$ calculé à l'aide de la droite de régression linéaire est donné par la formule ci-dessous :

$$
y=a+b x_{0} \pm t(\alpha / 2, n-2) S_{r} \sqrt{\frac{1}{n}+\frac{\left(x_{0}-\bar{x}\right)^{2}}{\sum\left(x_{i}-\bar{x}\right)^{2}}}
$$

- $\quad b$ et $a$ sont respectivement le coefficient directeur et l'ordonnée à l'origine de la droite de régression.

- $\quad s_{r}$ est l'écart-type résiduel.

Les valeurs expérimentales des masses de résidu sont ici notées $x$.

- $\quad n$ est la taille de l'échantillon (56).

- $\quad t$ valeur de la table de Student $;(\alpha / 2 ; n-2)=t(0,025 ; 54)=2,009$.

L'incertitude sur chaque valeur de $f$ est prise en compte de manière globale dans le calcul statistique par la variabilité résiduelle. En revanche, l'incertitude de chaque point expérimental $\left(x_{0}\right)$ n'a pu être évaluée puisqu'il n'a pas été techniquement possible d'effectuer plus d'une mesure de masse pour chaque point.

Les « courbes limites » sont tracées sur la figure 2 à partir des valeurs calculées par la formule (2) pour chaque valeur de $x_{0}$ reportées dans le tableau I. Ce tableau fait aussi apparaître l'étendue de l'intervalle de confiance et les incertitudes absolues et relatives pour chaque valeur de $x_{0}$. 
TABLEAU I

Détermination de l'intervalle de confiance et des incertitudes absolue et relative pour différentes valeurs de masse de résidu $x_{0}$ comprise entre 0 et $105 \mathrm{mg}$.

Interval of acceptation and absolute and relative uncertainties determination for different mass values $x_{0}$ between 0 and $105 \mathrm{mg}$.

\begin{tabular}{|c|c|c|c|c|c|}
\hline $\begin{array}{l}\text { Valeurs de } x_{0} \\
\quad(\mathrm{mg})\end{array}$ & $\begin{array}{l}\text { Valeurs de } f(y) \\
\text { selon la droite de } \\
\text { régression }\end{array}$ & $\begin{array}{c}\text { Borne } \\
\text { supérieure de } \\
\text { l'intervalle de confiance }\end{array}$ & $\begin{array}{c}\text { Borne } \\
\text { inférieure de l'intervalle } \\
\text { de confiance }\end{array}$ & $\begin{array}{l}\text { Incertitude } \\
\text { absolue }\end{array}$ & $\begin{array}{l}\text { Incertitude } \\
\text { relative }\end{array}$ \\
\hline 0 & 1,281319438 & 1,41138527 & 1,151253606 & 0,130065832 & 0,101509295 \\
\hline 5 & 1,407751063 & 1,527398777 & 1,288103349 & 0,119647714 & 0,084992096 \\
\hline 10 & 1,534182689 & 1,64407988 & 1,424285497 & 0,109897191 & 0,071632402 \\
\hline 15 & 1,660614314 & 1,761622097 & 1,559606531 & 0,101007783 & 0,060825552 \\
\hline 20 & 1,787045939 & 1,880272085 & 1,693819793 & 0,093226146 & 0,052167739 \\
\hline 25 & 1,913477565 & 2,000328119 & 1,82662701 & 0,086850554 & 0,045388854 \\
\hline 30 & 2,03990919 & 2,122117983 & 1,957700397 & 0,082208793 & 0,040300222 \\
\hline 35 & 2,166340815 & 2,245945558 & 2,086736073 & 0,079604742 & 0,036746177 \\
\hline 40 & 2,29277244 & 2,372011994 & 2,213532887 & 0,079239553 & 0,034560583 \\
\hline 45 & 2,419204066 & 2,500347525 & 2,338060606 & 0,081143459 & 0,033541387 \\
\hline 50 & 2,545635691 & 2,630800107 & 2,460471276 & 0,085164415 & 0,033455068 \\
\hline 55 & 2,672067316 & 2,763089603 & 2,581045029 & 0,091022287 & 0,034064369 \\
\hline 60 & 2,798498942 & 2,896888465 & 2,700109418 & 0,098389523 & 0,035157963 \\
\hline 65 & 2,924930567 & 3,031885241 & 2,817975893 & 0,106954674 & 0,036566568 \\
\hline 70 & 3,051362192 & 3,167815911 & 2,934908473 & 0,116453719 & 0,038164502 \\
\hline 75 & 3,177793818 & 3,304470562 & 3,051117074 & 0,126676744 & 0,03986311 \\
\hline 80 & 3,304225443 & 3,44168776 & 3,166763125 & 0,137462318 & 0,041601979 \\
\hline 85 & 3,430657068 & 3,579345139 & 3,281968997 & 0,148688071 & 0,043340989 \\
\hline 90 & 3,557088693 & 3,717350225 & 3,396827162 & 0,160261532 & 0,045054129 \\
\hline 95 & 3,683520319 & 3,85563289 & 3,511407748 & 0,172112571 & 0,046725023 \\
\hline 100 & 3,809951944 & 3,994139561 & 3,625764327 & 0,184187617 & 0,048343816 \\
\hline 105 & 3,936383569 & 4,132828935 & 3,739938204 & 0,196445365 & 0,049905036 \\
\hline
\end{tabular}


L'incertitude relative maximale est atteinte pour la valeur $x_{0}=0(10,15 \%$ arrondie à la valeur supérieure, soit $11 \%$ ). Afin de simplifier les calculs d'incertitudes lors du rendu des résultats, cette valeur d'incertitude relative qui correspond à un niveau de probabilité de $95 \%(k=2)$ sera appliquée à l'ensemble des valeurs de $f$ calculées à partir de la régression linéaire, quelle que soit la valeur de la masse de résidu $\mathrm{m}$ comprise entre 0 et $100 \mathrm{mg}$.

Remarque 1. L'incertitude sur l'activité tient compte entre autre de l'incertitude sur le facteur $f$, qui reste par retour d'expérience toujours bien inférieure à l'incertitude sur la mesure (de l'ordre de 20 à $30 \%$ ).

Remarque 2. La relation établie (coefficients $a$ et $b$ de la droite) a été validée à l'occasion de comparaisons inter-laboratoires. Elle est applicable dans nos conditions opératoires et ne peut être utilisée, par exemple, si la géométrie de comptage est différente de celle de notre appareil de mesure.

\section{Application à la surveillance de sites spécifiques de la défense}

La validation de l'expression de $f$ en fonction de la masse de résidus nous permet de déterminer l'indice de radioactivité alpha dans les eaux de sites de la défense où existe un risque de pollution par des radionucléides émetteurs alpha. C'est le cas des sites d'expérimentation des obus flèches à l'uranium appauvri.

\subsection{Les sites d'expérimentation d'obus flèches à l'uranium appauvri}

En France, il existe deux centres d'expérimentation d'obus flèches à l'uranium appauvri : le Centre d'étude de Gramat (CEG) et l'Établissement technique de Bourges (ETBS). Ces deux sites relèvent de la Délégation générale pour l'armement (DGA).

À Gramat, les tirs se font dans une enceinte fermée étanche composée d'une coupole et d'un tunnel de tir. Ils comprennent des tirs d'obus en uranium appauvri sur réceptacle dur (blindage ou matériau composite) et des tirs de charge à revêtement d'uranium. Ils permettent en outre une caractérisation des alliages d'uranium au moyen d'un lanceur à poudre ou de générateur d'ondes plates.

À Bourges, les tirs sont effectués en extérieur pour déterminer la résistance mécanique du projectile ou pour évaluer les caractéristiques de pénétration en balistique terminale sur cible inerte. La flèche est stoppée par du sable dans un réceptacle aménagé.

Dans les deux cas, les tirs génèrent des poussières d'uranium qui se déposent d'abord sur le sol qui peuvent ensuite contaminer l'eau des nappes phréatiques. 
TABLEAU II

Caractéristiques radioactives des isotopes de l'uranium constitutifs de l'uranium appauvri. Radioactive characteristics of uranium isotopes present in depleted uranium.

\begin{tabular}{|c|c|c|c|c|c|c|}
\hline \multirow{2}{*}{$\begin{array}{c}\text { Isotopes } \\
\text { Périodes }(T)\end{array}$} & \multicolumn{2}{|c|}{ Rayonnements X } & \multicolumn{2}{|c|}{ Rayonnements gamma } & \multicolumn{2}{|c|}{ Rayonnements alpha } \\
\hline & $\begin{array}{c}\text { Énergie } \\
(\mathrm{keV})\end{array}$ & $\% *$ & $\begin{array}{c}\text { Énergie } \\
(\mathrm{keV})\end{array}$ & $\% *$ & $\begin{array}{c}\text { Énergie } \\
(\mathrm{MeV})\end{array}$ & $\% *$ \\
\hline$T=2,44 \times 10^{5} \mathrm{a}$ & & & $\begin{array}{c}53,3 \\
120,9\end{array}$ & $\begin{array}{l}0,68 \\
0,23\end{array}$ & $\begin{array}{l}4,723 \\
4,775\end{array}$ & $\begin{array}{l}28,4 \\
71,4\end{array}$ \\
\hline$T=7,98 \times 10^{8} \mathrm{a}$ & $\begin{array}{c}15,5 \\
89,9 \\
93,3 \\
105\end{array}$ & $\begin{array}{c}29 \\
2,7 \\
4,4 \\
2\end{array}$ & $\begin{array}{l}109,16 \\
143,76 \\
163,33 \\
185,72 \\
205,31\end{array}$ & $\begin{array}{c}1,5 \\
10,9 \\
5,0 \\
57,4 \\
5,01\end{array}$ & $\begin{array}{l}4,218 \\
4,365 \\
4,400 \\
4,556 \\
4,599\end{array}$ & $\begin{array}{c}5,7 \\
17,0 \\
55,0 \\
4,2 \\
5,0\end{array}$ \\
\hline$T=4,47 \times 10^{9} \mathrm{a}$ & 15,4 & 8,7 & $\begin{array}{c}49,55 \\
63,29 * * \\
92,38 * * \\
92,80 * *\end{array}$ & $\begin{array}{c}0,064 \\
4,5 \\
2,6 \\
2,6\end{array}$ & $\begin{array}{l}4,149 \\
4,196\end{array}$ & $\begin{array}{l}23 \\
77\end{array}$ \\
\hline
\end{tabular}

* : pourcentage d'émission ou intensité rapportée à 100 transformations nucléaires.

** : énergie de ${ }^{234 \mathrm{~m}} \mathrm{Th}$, descendant de l'uranium, de période 24 jours.

\section{TABLEAU III}

Composition isotopique en masse $(\%)$ et en activité alpha $(\%)$ de différents types d'uranium (naturel, appauvri et enrichi).

Isotopic composition in mass $(\%)$ and in alpha activity $(\%)$ of uranium different types (natural, depleted and enriched).

\begin{tabular}{|c|c|c|c|c|}
\hline & & Uranium naturel & $\begin{array}{l}\text { Uranium appauvri } \\
\text { à } 0,3 \% \text { en }{ }^{235} U\end{array}$ & $\begin{array}{l}\text { Uranium enrichi } \\
\text { à } 3 \% \text { en }{ }^{235} U\end{array}$ \\
\hline \multirow{3}{*}{ 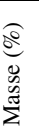 } & ${ }^{234} \mathrm{U}$ & 0,055 & 0,02 & $<0,1$ \\
\hline & ${ }^{235} \mathrm{U}$ & 0,72 & 0,3 & 3,27 \\
\hline & ${ }^{238} \mathrm{U}$ & 99,28 & 99,68 & 96,73 \\
\hline \multirow{3}{*}{ 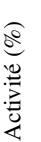 } & ${ }^{234} \mathrm{U}$ & 49 & 19 & 80 \\
\hline & ${ }^{235} \mathrm{U}$ & 2 & 2 & 4 \\
\hline & ${ }^{238} \mathrm{U}$ & 49 & 79 & 16 \\
\hline
\end{tabular}

\subsection{Données générales sur l'uranium appauvri}

L'uranium naturel est composé des isotopes 238, 234 et 235 de l'uranium, tous émetteurs alpha ainsi que de leurs descendants émetteurs alpha, bêta ou gamma (Le Guen et Bérard, 1998). Leurs caractéristiques radiologiques sont données dans le tableau II.

Les proportions respectives de ces isotopes peuvent être modifiées pour aboutir à de l'uranium enrichi en ${ }^{235} \mathrm{U}$ (utilisable comme combustible) ou appauvri en ${ }^{235} \mathrm{U}$ (sous-produit de l'industrie nucléaire). La composition isotopique des différents types d'uranium est détaillée dans le tableau III. 
La toxicité de l'uranium est liée à ses caractéristiques chimiques et/ou radiologiques. En fonction du mode de contamination (ingestion ou inhalation), de la forme chimique impliquée (soluble ou insoluble), du taux d'enrichissement ou encore du type d'exposition (aiguë ou chronique), l'un de ces modes de toxicité peut prendre le pas sur l'autre (Yuile, 1973 ; Chalabreysse et al., 1989).

La toxicité chimique est comparable à celle des métaux lourds (cadmium, mercure, plomb, ...) et les reins sont particulièrement sensibles. La toxicité radiologique ne peut être véritablement retenue que dans le cas de l'uranium enrichi. Elle dépend essentiellement de la quantité incorporée et la dose estimée dépendra d'autres paramètres (forme physico-chimique, teneurs en impuretés, ...).

De par sa densité, l'uranium appauvri sert notamment à la fabrication d'obus flèches et comme blindage dans les chars. L'uranium appauvri étant composé de radio-isotopes émetteurs de particules alpha, la mesure de la radioactivité alpha globale de l'eau telle qu'elle est définie dans le code de la santé publique est suffisante pour assurer la surveillance des sites concernés vis-à-vis de ce risque particulier. C'est le cas à Bourges et à Gramat.

\subsection{Interprétation des résultats}

Une variation anomalique de l'activité alpha d'un site ne signifie par pour autant qu'il y eu pollution par l'uranium appauvri. En effet, les causes de variations sont multiples (fluctuation de la teneur en radon et de ses descendants, augmentation de la charge en matière en suspension qui peuvent relarguer des éléments naturels émetteurs alpha, ...) et elle ne préjuge donc pas des radionucléides dont l'activité a pu spécifiquement évoluer. En pratique, face à une telle situation, le laboratoire effectue sur l'échantillon « source » une spectrométrie à l'aide d'un spectromètre alpha dit à « chambre à grille ». Le relativement bon rendement (de l'ordre de $50 \%$ ) obtenu avec cet appareil doit théoriquement nous orienter vers le radionucléide à l'origine d'un indice alpha plus élevé. Si un doute persiste, un dosage spécifique des isotopes de l'uranium dans la matrice est effectué, complété par des mesures sur de nouveaux prélèvements. L'identification et la quantification des radio-isotopes peuvent encore être effectuées conformément à l'arrêté du 17 septembre 2003 (JORF, 2003b).

\section{Conclusion}

L'indice de radioactivité alpha dans une eau est un des quatre paramètres introduits par l'OMS pour la surveillance de la qualité radiologique des eaux destinées à la consommation humaine. La méthode de mesure utilisée par notre laboratoire est normalisée. Elle nécessite d'établir au préalable une relation entre la masse de 
résidus d'évaporation de l'échantillon et le facteur de correction de l'auto absorption des particules alpha $(f)$. Un calcul d'activité sans tenir compte de ce facteur aurait des répercussions sur les résultats (sous estimation) et sur l'interprétation sanitaire. Le mode opératoire expérimental utilisé a été décrit. Il a permis d'obtenir une relation linéaire de type $f=0,0253 m+1,2813$. Ce modèle mathématique a été validé statistiquement et une incertitude relative pour chaque masse a pu être établie à $11 \%(k=2)$. Cette relation s'applique à des échantillons d'eau présentant des caractéristiques physico-chimiques similaires à celles des échantillons ayant permis sa détermination et pour une gamme de masse de résidus s'étendant jusqu'à 100 mg.

La contamination de l'eau par de l'uranium appauvri, élément radioactif émetteur alpha, doit être envisagée dans l'environnement des sites où ce matériau est mis en œuvre. Les sites d'expérimentation de la défense où sont effectués les tirs d'obus flèches à l'uranium appauvri ont été présentés.

\section{RÉFÉRENCES}

Bouyer J. (2000) Méthodes statistiques (médecine-Biologie). INSERM, 351p.

Chalabreysse J., Beau P., Chevalier C., Jeanmaire L., Bataller G., Berard P., Gilbert B. (1989) French experience with uranium compounds, Radiat. Prot. Dosim. 26, 49-56.

CSP (2002) Code de la santé publique, livre 3, protection de la santé et de l'environnement. Au titre des eaux, titre 2 - Sécurité sanitaire des eaux et des aliments, Chapitre 1 - Eaux potables - section 1 - eaux destinées à la consommation humaine à l'exclusion des eaux minérales naturelles.

Gérasimo P., Laroche P. (2004) Surveillance radiologique des eaux de boissons, Rev. Fr. Lab. 363, 2532.

JOEC (1998) Directive 98/83/CE du conseil du 3 novembre 1998 relative à la qualité des eaux destinées à la consommation humaine, JOEC du 5 décembre 1998.

JORF (2003a) Arrêté du $1^{\text {er }}$ septembre 2003 définissant les modalités de calcul des doses efficaces et des doses équivalentes résultant de l'exposition des personnes aux rayonnements ionisants, JORF du 13 novembre 2003.

JORF (2003b) Arrêté du 17 septembre 2003 relatif aux méthodes d'analyse des échantillons d'eau et à leurs caractéristiques de performance, JORF du 30 octobre 2003.

JORF (2004) Arrêté du 12 mai 2004 fixant les modalités de contrôle de la qualité radiologique des eaux destinées à la consommation humaine, JORF du 18 juin 2004.

Kleinschmidt Ross I. (2004) Gross alpha and beta activity analysis in water-a routine laboratory method using liquid scintillation analysis, Applied Radiation and Isotopes 61, 333-338.

Le Guen B., Bérard P. (1998) Uranium et ses composés, Encycl. Méd. Chir. Elsevier, Paris. Toxicologie-Pathologie professionnelle, 16-008-U-10, 6p.

NF (2004) Norme NF M 60-801, septembre 2004, Mesure de la radioactivité dans l'environnement Eau - Mesurage de l'indice de radioactivité alpha en équivalent plutonium dans l'eau peu chargée en sels.

Parsa B., Obed R.N., Nemeth W.K., Suozzo G.P. (2005) Determination of gross alpha, ${ }^{224}$ Ra, ${ }^{226}$ Ra, and ${ }^{228} \mathrm{Ra}$ activities in drinking water using a single sample preparation procedure, Health Phys. 89, 660-666. 
Souvey P. (2002) Savoir utiliser la statistique - Outil d'aide à la décision et à l'amélioration de la qualité, AFNOR.

Toscheva Z., Stoyanova K., Nikolchev L. (2004) Comparisons of different methods for uranium determination in water, J. Environm. Rad. 72, 47-55.

WHO (2004) Guidelines for drinking-water quality, volume 1: recommendations, 3rd edn. World Health Organization, Geneva.

Yuile Cl. (1973) Animals experiments, in: H.C. Hodge, J.N. Stannard, J.B. Hursch, Eds. Hanbook of experimental pharmacology, Vol. 36. Springer-Verlag, New York. 